\title{
Heat transport in phononic-like membranes: modeling and comparison with modulated nano-wires
}

\author{
Maxime Verdier ${ }^{1}$, David Lacroix ${ }^{2}$, Konstantinos Termentzidis ${ }^{3}$ \\ Université de Lorraine, LEMTA, UMR 7563, Vandœuvre-lès-Nancy ,F-54500, France \\ CNRS, LEMTA, UMR 7563, Vandoeuvre-lès-Nancy ,F-54500, France
}

\begin{abstract}
The thermal conductivity of phononic-like membranes and their analogous nanowires are computed with both Molecular Dynamics and Monte Carlo simulations. Using both methods, the thermal conductivity of the porous membranes with cylindrical pores is found to be lower than the nano-wire's one for the same scattering surface to volume. This work confirms experimental observations by Nomura et al [1] and Yu et al [2] for similar systems. The lower thermal conductivity of membranes is attributed to the different orientation of the scattering surfaces either at the pores or at free surfaces and not to coherent effects. The comparison between the porous membranes and nano-wires with periodic cylindrical modulations shows that thermal transport is hindered further when there are surfaces perpendicular to the heat flow. These results show that coherent effects have no impact on thermal transport in both kind of nano-structures at room temperature.
\end{abstract}

Keywords: Nano-structuration, Silicon, Molecular Dynamics, Monte Carlo, thermal conductivity

\footnotetext{
${ }^{1}$ maxime.verdier@univ-lorraine.fr

2 david.lacroix@univ-lorraine.fr

${ }^{3}$ konstantinos.termentzidis@univ-lorraine.fr
} 


\section{Introduction}

During the last decades, increased interest has been observed to tailor different physical properties of nano-structures and nano-structured materials. Unique characteristics and effects are found in nano-structured semi-conductors 5 compared to their bulk properties. Silicon is the most common semi-conductor on Earth and is a non-pollutant material. Therefore, nano-structured Silicon has been intensively studied experimentally and theoretically for its optical 3, 4] or thermal properties [5, 6, 7] among a plethora of other properties. The importance of thermal management of nano-structures has arisen as the electronic devices became smaller and more powerful. Nano-structured semi-conductors are widely used for energy thermoelectric conversion [8, 9] or in photovoltaic devices [10].

Efficiency of thermoelectric materials depends on their electrical to thermal conductivities ratio (figure of merit), thus the thermal conductivity (TC) has

15 to be very low. However, current thermoelectric materials have a poor figure of merit and cannot be massively used for energy production. Then, the aim of the nano-structuration of semi-conductors is often to reduce the thermal transport by multilength scale hierarchical structuring with purpose to increase the density of interface or introducing point or extended defects. On the other hand as electrons have a much lower mean free path than phonons 11, it can be assumed that their conductivity is preserved in most of the nano-structured semi-conductors.

Historically, the study of heat transfer in composite materials began with Maxwell [12] and Lord Rayleigh [13] at the end of the $19^{\text {th }}$ century. There25 after, thermal transport in nano-structured compounds gained interest, including porous and nano-porous materials [14, 15, 16. Different kinds of nanostructuration are possible today, especially with the fast development of fabrication methods. The TC of super-lattices alternating layers of different semiconductors have been intensively investigated during the last decade [17, 18, 19].

30 In such structures, band folding plays a key role in TC lowering. The reduc- 
tion of the thermal transport was also observed in thin films $20,21,22,23$. and nano-wires $[24,25,26,27,28,29$. In the recent years, several studies are devoted to thermal behavior of bulk nano-porous systems [30, 31, 32, 33, or nano-porous membranes [34, 35, 36, 37, 38.

Recently, a new method has been proposed to reduce thermal transport in periodic nano-structures. By analogy to the photonic crystals, which are also known as photonic band gap materials as there are forbidden photon propagation frequencies [39, 40, an artificially and periodically structured material could exclude phonons of certain frequencies [41, 36, 42, 43, 44]. These phonon band gaps are due to scattering and interference phenomena, the latter being known as coherent effects. If the prohibited phonon modes are heat carriers, the TC would be further reduced [45. Abnormally low TCs have been experimentally observed in periodically nano-structured Silicon [2] and this was attributed to these coherent effects.

Nomura et al compared the experimental TCs of membranes with periodic cylindrical pores to those of nano-wires [1]. They found that thermal transport is weaker in membranes, despite their low scattering surface to volume ratio compared to nano-wires with similar characteristic size and features. This might be due to phononic effects (i.e. any effect related to a modification of phonon 50 dispersion properties like band flattening, band gap ... or coherent scattering of phonon due to the periodicity of the hole pattern), but authors mentioned that the "local angles between local and global heat flux" could be responsible of this phenomenon 11. In other words, they point out the different impact of scattering surfaces on thermal transport according to their spatial orientation compared to the direction of the main thermal gradient. At this stage a simple schematic description of phonon transport can be depicted. When the TC is measured along the growth direction of a nano-wire, all scattering surfaces of the system are parallel to the heat flux. If phonons are diffusely scattered at the free surfaces, half of them are back-scattered (Diffuse Mismatch Model). In 60 contrast, in the "phononic crystal" (hollow membrane) the surface of the pore (which is here a part of the scattering surfaces with upper and lower boundaries) 
is mainly perpendicular to the heat flux (see fig. 11 ). Then, the back-scattering of phonons in the membrane is increased as compared to the nano-wire and the TC of the membrane is reduced.

In this work, the same strategy as Nomura et al has been exploited, making the comparison between porous membranes and nano-wires with two numerical techniques: Monte Carlo solving of the BTE and Molecular Dynamics. The comparison between the two nano-structured materials shows the importance of the orientation of the scattering surfaces on thermal transport. Technical details about the computations of the $\mathrm{TC}$ with these two methods are presented in section 2, then the geometries of the modeled systems are defined in section 3. TC experimental results and numerical simulation results are compared in section 4 . In the same section, the phonon densities of states computed with Molecular Dynamics are presented to give physical insights. Finally, in section

755 modulated nano-wires are modeled and their TC is given in comparison to membrane's TC with the same geometric parameters.

\section{Simulation methods}

\subsection{Molecular Dynamics}

Molecular Dynamics simulations are performed with LAMMPS [46]. Equiso librium Molecular Dynamics (EMD) method is used to determine the TC via the Green-Kubo formula [4]:

$$
k_{x y}=\frac{\mathcal{V}}{k_{B} T^{2}} \int_{0}^{+\infty}\left\langle J_{x}(t) J_{y}(0)\right\rangle d t
$$

where $x$ and $y$ subscripts denote space directions, $\mathcal{V}$ is the system's volume, $J$ is the heat flux, $t$ is is the time, $T$ is the system's temperature and $k_{B}$ the Boltzmann constant. Then, the TC can be obtained for all directions. For membranes, the flux auto-correlation function is calculated in $x$ and $y$ directions $\left(J_{x}(t) J_{x}(0)\right.$ and $J_{y}(t) J_{y}(0)$, respectively). The in-plane TC is the average of the two thermal conductivities in direction $x$ and $y$ (see fig. 17). For nano-wires, 
the flux auto-correlation function is calculated only for $x$ direction which is the growth direction of nano-wires (see fig. 1p). The interactions between the atoms are described with the Stillinger-Weber potential [48] with modified coefficients by Vink et al [49] as this latest parameterization is able to describe thermal transport in Silicon, in both crystalline and amorphous phases.

The time step is set to $0.5 \mathrm{fs}$ in all EMD runs. At the beginning of the simulation, the system is relaxed at $T=300 \mathrm{~K}$ with a NVT ensemble during 200 ps. Then the computation of the TC is performed every 40 ps under a NVT ensemble, estimating the flux fluctuations correlation every $10 \mathrm{fs}$, for a total duration of $10 \mathrm{~ns}$. The TC is averaged over the second half of the simulation, when the steady state is established, in order to reduce the uncertainty on the results [50]. Moreover, each simulation is repeated 10 times with different seeds and the TC is averaged. Periodic boundary conditions are applied on surfaces perpendicular to the directions of interest (nanowire's growth direction, membrane's $x$ and $y$ directions), while non-periodic and shrink-wrapped boundary conditions are applied on the free surfaces. Size effects, i.e. influence of the simulation box size, have been considered by testing the model on cubic box with edge length of $5,10,15$ and $20 a_{0}$. For these cases mean value of bulk TC remains the same, only the uncertainty increases when the box gets smaller. In the bulk crystalline Silicon case, the TC is estimated to be $k=164 \pm 21 \mathrm{~W} / \mathrm{m} \mathrm{K}$. That is in good agreement with experimental values [51, 52].

With Molecular Dynamics, the phonon's Density of States (DOS) of the system can also be extracted from the trajectories of atoms. Indeed, the displacement of atoms can be decomposed by Fourier transform to extract the density of frequencies of phonons. In this work, the specden plugin of the $V M D$ Signal Processing Plugin Package [53] was used to calculate the phonon DOS of the porous membranes.

\subsection{Monte Carlo}

The Monte Carlo technique is based on the resolution of the Boltzmann Transport Equation (BTE) in the relaxation time approximation (eq. 2] [33, 24, 
where $f$ is the phonon's distribution function, $v_{g}$ is the group velocity, $\tau$ is the relaxation time, while exponant "0" denotes thermal equilibrium:

$$
\frac{\partial f}{\partial t}+\overrightarrow{v_{g}} \cdot \overrightarrow{\nabla f}=\frac{f^{0}-f}{\tau(\omega, T)}
$$

In this approach, phonons are considered as particles with wave properties (pulsation, group velocity and polarization) and their displacements and interactions are modeled through several steps:

- Design of the geometry of the structure. Parameters such as size and boundary conditions are defined, then the system is divided into 20 cells in the $x$ direction.

- Initialization of phonon population with random space positions, determining their characteristics according to the spectral properties of the medium (Bose-Einstein distribution, dispersion curves and density of states). These properties are those of the bulk crystalline Silicon, so coherent phononic effects cannot appear in the results obtained with Monte Carlo. A weak temperature gradient is applied in the $x$ direction by initializing more phonons in the first cell (Bose-Einstein distribution at higher temperature).

- Drift of the phonons during the time step, knowing their initial position and their group velocity. This step is represented by the advective term $\overrightarrow{v_{g}} \cdot \overrightarrow{\nabla f}$ in the BTE. Reflections at the boundaries and the pores are achieved during this step. For membranes specular reflection (periodic boundary) is assumed in the $y$ direction, whereas diffuse reflection is set in the $z$ direction (see fig. 17). For nano-wires, reflection at the edge of a cell are both diffuse in $y$ and $z$ directions (see fig. 1 $\mathrm{b}$ ). The reflection at the pores can be set to entirely specular or purely diffuse.

- Collisions of the phonons during the time step. This step is represented by the right hand side term of the BTE $\left(f^{0}-f\right) / \tau(\omega, T)$ and brings the system back to equilibrium after the drift phase by modifying the phonon's 
properties. It takes into account the phonon scattering due to impurities and anharmonic processes (phonon-phonon interactions) thanks to the semi-empirical lifetime of Holland [54]. Thus, the Normal and Umklapp processes are differentiated.

- Thermalization of the first and last cells according to the temperature gradient. All the phonons in these thermostats are deleted then replaced by new ones in order to impose the hot and cold temperatures of the gradient.

During MC simulation, the three last steps are repeated for a total duration of about 200 ns. A steady state is usually reached after tens of nanoseconds (depending on the thermal resistance), when the temperature gradient becomes linear between the two thermostats. The heat flux and the temperature of each cell during the steady state are computed from the properties of phonons (pulsation and velocity) at every time step. Finally, temperatures and flux are averaged over the steady state and the TC is extracted according to the Fourier's law (eq. 3 with $S$ the cross-section). The code which performed all these steps was developed by our work team (more details can be found in refs [55, 33, 24 and was validated by retrieving the bulk crystalline Silicon conductivity: $k \simeq 148 \mathrm{~W} / \mathrm{m} \mathrm{K}$. In this work, the typical time step is $\Delta t=1 \mathrm{ps}$ and the temperature gradient is set from 299 to $301 \mathrm{~K}$ for structures with characteristic length $L_{x}$ of $4800 \mathrm{~nm}$.

$$
\frac{\vec{J}}{S}=-k \times \vec{\nabla} T
$$

\section{Modeled systems}

Three nano-scale geometries of Silicon are modeled in the current work:

- phononic-like nano-porous membranes (np-memb)

- nano-wires with constant cross-section (NW)

- nano-wires with modulated cross-section (m-NW) 
As in ref [1, membranes with cylindrical nano-pores (np-memb) are compared to nano-wires (NW) with dimensions adjusted in a way that the nanowires just fit in the space between the pores of the membranes (fig. 1 $1 \mathrm{a}$ and 1b). So the width of a nano-wire is equal to the neck $n=a-d$ with $a$ the 175 periodicity of the equivalent porous membrane and $d$ the diameter of the pores. Both in-plane TC of membranes and TC of NWs are considered along the $x$ direction. Due to the lower material volume and the greater scattering surface of the NW compared to the membrane, it was expected that the NW has lower TC than the membrane. But the opposite behavior was observed by Nomura et al [1] and $\mathrm{Yu}$ et al 2]: the NW has a greater TC than the membrane. Both of these two works [1, 2] were based on experimental TC measurements. The current work confirms this behavior numerically.

The two geometries have been modeled with both numerical methods (Molecular Dynamics and Monte Carlo) but with different characteristic sizes. With Molecular Dynamics, the thickness of the membrane is kept constant at $e=$ $15 a_{0}=8.145 \mathrm{~nm}$, the diameter of the pores is set to $d=10 a_{0}=5.43 \mathrm{~nm}$ while the periodicity of the membrane varies between 15 and $34 a_{0}$ (8.145 and $18.5 \mathrm{~nm}$, respectively) with $a_{0} \simeq 5.43 \AA$ the lattice constant. Thus, the equivalent NW's height is always $15 a_{0}$ and its width varies between 5 and $24 a_{0}$ 190 (neck between the pores of the membrane). With Monte Carlo, the periodicity and the thickness of the membrane are respectively set to $a=240 \mathrm{~nm}$ and $e=145 \mathrm{~nm}$ (comparable to the experimentally fabricated membranes of Nomura et al), while the diameter $d$ of the pores varies between 0 and $220 \mathrm{~nm}$ (zero corresponds to membranes without pores). Thus, the height of the equivalent NW is kept at $e=145 \mathrm{~nm}$ and its width varies between 220 and $0 \mathrm{~nm}$. We have chosen different sets of values for the two methods because of the limitations due to computational cost for MD on one hand and the validity scale of $\mathrm{MC}$ on the other hand, keeping a similar ratio $n / e$ for both methods.

A third equivalent system has been modeled (fig. 11). It is a modulated 200 nano-wire $(\mathrm{m}-\mathrm{NW})$ where hollows with the shape of a half-cylinder are periodically set along the $x$ axis (length of the m-NW) at both ends of the structure in 
the $y$ direction. In order to compare the new system with the porous membrane, the half-cylinders have the same diameter $d$ as the pores, while the maximum width of the m-NW is equal to the periodicity of the membrane. Thus, the

\subsection{Molecular Dynamics}

The TCs obtained with means of Molecular Dynamics are summarized in table 1 The ratio of scattering surface $S$ to volume $\mathcal{V}$ is provided for each configuration as it usually controls the $\mathrm{TC}$ of isotropic and homogeneous porous

minimum width in the narrow part of the $\mathrm{m}-\mathrm{NW}$ is equal to the neck of the membrane. The TC is always computed along the $x$ axis. Now the only difference between this latest configuration and the phononic-like membrane is the boundary condition along $y$ axis: periodic for the membrane and fixed for the m-NW.

To summarize we study three different systems; i.e. a phononic membrane, a nanowire and a modulated nanowire. In phononic membranes it has already be demonstrated [1, 57] that when energy carrier MFPs become smaller than a characteristic size which is related with the nanostructuration geometry, then the $\mathrm{TC}$ is affected. This characteristic size can be: i) the surface to volume ratio when boundaries dominate the transport, or ii) the neck of the np-memb when the distance between holes is very small[57]. In the first case $(L=S / \mathcal{V})$, such behavior can be explained in the frame of the Casimir limit theory [58] which well describe thin films and nanowires. In the second case $(L=n)$, the characteristic length is the neck of the nanostructure and physic of transport is ruled by the constriction effect also known as "ballistic transport" [59, 60. In the latter case, one can refer to studies dealing with modulated and constricted nanowires [24] that exhibit similar features. Hereafter, we are going to investigate the TC of the three systems and try to see if the suggested mechanisms rules effectively heat transport in np-memb.

\section{Comparison between phononic-like membranes and nano-wires}


4). In spite of its lower $S / \mathcal{V}$ ratio, the $\mathrm{TC}$ of the membrane is systematically lower than NW's one, as previously experimentally observed by Yu and Nomura 2, 1]. As Molecular Dynamics are a numerical technique, this observation does not come from experimental uncertainty or exterior perturbations.

Table 1: Comparison between the TCs of porous membranes and equivalent NWs. The $S / \mathcal{V}$ ratio is given for each configuration. The TC of the membrane is systematically lower, despite its lower $S / \mathcal{V}$ ratio.

\begin{tabular}{|c|c|c|c|c|}
\hline \multirow{2}{*}{$\begin{array}{c}\text { Neck } n \\
\left(a_{0}\right)\end{array}$} & \multicolumn{2}{|c|}{$k(\mathrm{~W} / \mathrm{m} \mathrm{K})$} & \multicolumn{2}{c|}{$S / \mathcal{V}\left(\mathrm{nm}^{-1}\right)$} \\
\cline { 2 - 5 } & membrane & nano-wire & membrane & nano-wire \\
\hline 5 & $3.4 \pm 0.3$ & $14.0 \pm 1.9$ & 0.64 & 0.98 \\
\hline 10 & $8.6 \pm 0.5$ & $22.1 \pm 2.3$ & 0.43 & 0.61 \\
\hline 15 & $15.2 \pm 1.9$ & $26.7 \pm 2.7$ & 0.35 & 0.49 \\
\hline 24 & $22.7 \pm 2.0$ & $32.8 \pm 3.3$ & 0.16 & 0.22 \\
\hline
\end{tabular}

The reduction of the $\mathrm{TC}$ of the membranes compared to their equivalent NW ranges from 30 to $75 \%$ depending on the "neck" size. This reduction is more pronounced when the neck is small. In figure 2 , the $\mathrm{TC}$ of both systems is plotted in terms of neck size. The ratio $k_{N W} / k_{n p-m e m b}$ (blue crosses, right blue axis) decreases when the neck size increases. We assume that for neck sizes

$$
\frac{S}{\mathcal{V}}=\left\{\begin{array}{cc}
\frac{\pi d e+2\left(a^{2}-\frac{\pi d^{2}}{4}\right)}{e a^{2}(1-\phi)} & \text { for a membrane } \\
\frac{2(e+n)}{e \times n} & \text { for the equivalent nano-wire }
\end{array}\right.
$$

larger than the phonon mean free path, the nano-wire's TC might be smaller than the membrane's one. Due to computational time issues we could not test this hypothesis with EMD.

Three assumptions can be considered to explain why the membrane's TC is much lower compared to NW's one:

1. Phononic effects might be dominant at low temperatures in the phononiclike membranes because of the periodicity of the porosity. Is this the case 
at room temperature? Band folding and band gap could occur in the dispersion curves and it would drastically lower the TC.

2. The effect of the orientation of the scattering surface is important, as supposed by Nomura [1] and demonstrated in ref [56]. In a NW, all scattering surfaces are parallel to the direction of measurement ( $x$ axis), while in membranes this surface is the combination of free surfaces (membrane's walls parallel to $x$ axis) and pore surfaces (curved surface, sometimes perpendicular or tangential to $x$ axis). The surfaces perpendicular to the direction of TC measurement could prevent the thermal transport more efficiently than the parallel surfaces (lateral surfaces of a NW).

3. In the simulation, it is possible that the roughness is more important for the pore surfaces, which are curved, than for the free surfaces, which are flat. A part of the phonons could be reflected specularly by free surfaces of the membrane while the pores would diffuse them. The NW having no pores, all its scattering surface would be partially specular and its TC would be greater than expected in purely diffusive case.

In figure 3 , the DOS of the three membranes are compared to the one of the membrane of same thickness without pores. No direct band gap due to the porosity is observed at $300 \mathrm{~K}$ and the modifications compared to the non-porous membrane are negligible except for the smaller system. In the latter, the TO and TA peaks are reduced. The TO polarized phonons contribute very little to the thermal transport in Silicon at room temperature [61, so the reduction of the TO peak should have no significant influence on the TC. However the TA polarized phonons are the main heat carriers, so the reduction in this peak could further lower the TC. This could explain why the reduction of the TC compared to the NW's one is so large for the membrane with the smallest periodicity. But for the two largest systems, the reduction in the TC does not seem to be due to phononic effects. 


\subsection{Monte Carlo}

The TCs calculated with Monte Carlo are plotted in the figure 4. For the membranes, two cases are considered: the reflection of phonons at the pore boundaries can be specular or diffuse. The TC is systematically lower when diffuse conditions are applied on the pore surface. Our geometric parameter values are based on the fabricated and measured membranes of Anufriev et al [57. Their results have been added in the figure for comparison (stars). The numerical values are in pretty good agreement with the experimental ones. Indeed, the Monte Carlo method underestimates the experimental TC but the trend is the same and the uncertainty on the measurement is unknown. Once more, the TC of the NW is greater than the membrane's one when the neck is small, regardless of the reflection condition on the pores. Two regimes are observed in the figure 4

- When the neck size is reduced (smaller than $150 \mathrm{~nm}$ ) because of the great diameter of the pore $(d>90 \mathrm{~nm})$, the TC of the NW is larger than the membrane's one.

- When the pore's diameter is small, the neck is large (greater than $150 \mathrm{~nm}$ ) and the $\mathrm{TC}$ is lower for the NW than in nano-porous membranes.

Molecular dynamics results and experimental values of refs [1, 2] belong without doubt in the first regime. Here, for the plotted Monte Carlo results, the crossing of the two curves occurs at a critical neck size $n_{c}=150 \mathrm{~nm}$. The periodicity of the membrane is $240 \mathrm{~nm}$, so the ratio $n_{c} / a=150 / 240$ is close to $2 / 3$. In order to check if this finding is general, a systematic study on the periodicity and the thickness of the membranes has been performed with Monte Carlo (2 thicknesses and 4 periodicities are depicted in figures added in supplementary data). For all studied cases the $n_{c}$ does not depend on the membrane thickness but only on the periodicity. Moreover, the crossing of the TC of the two nanostructures occurs when the neck size is roughly equal to $2 / 3$ of the periodicity (tab. 22). Corresponding graphs are given in supplementary materials section. 
Table 2: Critical neck size $n_{c}$ for different periodicities and thicknesses of membranes. When the neck is greater than $n_{c}$, the TC of the equivalent nano-wire is lower than the TC of the membrane. When the neck is less than $n_{c}$, thermal transport is further reduced in the membrane than in the nano-wire. The ratio $n_{c} / a$ is always close to $2 / 3$.

\begin{tabular}{|c|c|c|c|c|}
\hline \multirow{2}{*}{$\begin{array}{c}a \\
(\mathrm{~nm})\end{array}$} & \multicolumn{2}{|c|}{$e=145 \mathrm{~nm}(\mathrm{MC})$} & \multicolumn{2}{c|}{$e=80 \mathrm{~nm}(\mathrm{MC})$} \\
\cline { 2 - 5 } & $n_{c}(\mathrm{~nm})$ & $n_{c} / a$ & $n_{c}(\mathrm{~nm})$ & $n_{c} / a$ \\
\hline 160 & 100 & 0.625 & 105 & 0.66 \\
\hline 200 & 125 & 0.625 & 125 & 0.625 \\
\hline 240 & 150 & 0.625 & 160 & 0.67 \\
\hline 280 & 185 & 0.66 & 190 & 0.68 \\
\hline
\end{tabular}

The TC of the phononic-like membrane is about $60-80 \%$ of the NW's one cally diffuse. Concerning the pore's surfaces, the reflection type can be set to 
purely specular or diffuse at the beginning of the simulation. So, even with the pore condition set to specular and the exterior walls entirely diffuse, the porous membrane can have a TC lower than the NW for a same $S / \mathcal{V}$. So the third hypothesis of the section 4.1 can also be excluded. There is only left the second one, i.e. the surfaces of the pores, which are curved and partially perpendicular to the direction of measurement, are more efficient to reduce the thermal transport than the exterior surfaces, which are parallel to the $x$ axis. In order to evaluate this assumption, an other comparable system (third one depicted in section 3) has been modeled with Monte Carlo and Molecular Dynamics.

\section{Modulated nano-wires}

In this section, the TC of the third modeled system is investigated. If the orientation of the scattering surfaces is the main reason of the larger reduction of the TC in the porous membranes compared to the NWs without modulations, then the TC of the equivalent modulated NWs (m-NWs) with periodic and cylindrical modulations would always be less than or equal to the membrane's one. It is expected that the TC of the m-NW is much less than the membrane's one when $d$ is small because of the back-scattering which occurs on the free surface perpendicular to the $y$ direction only in the m-NW (the periodic conditions perpendicular to $y$ axis in the membrane do not reduce the thermal transport). When $d$ approaches to $a$, the impact of the $y$ boundary conditions on thermal transport is less important as reflections on the cylinders become more frequent than free surface scattering. So the TCs of the two systems should be the same. This is exactly what is observed in figure 6 .

With Monte Carlo, the m-NW has been modeled with dimensions comparable to those of the systems of the previous section 4.2 : the height of the $\mathrm{m}-\mathrm{NW}$ is equal to the thickness of the membrane $(e=145 \mathrm{~nm})$, its maximum width is $a=240 \mathrm{~nm}$ and the diameter $d$ of the cylinders varies between 0 and $220 \mathrm{~nm}$. For the membrane, the two cases of specular and diffuse reflections on the pores were investigated. In order to compare the new system to the mem- 
brane one, both reflection conditions on the cylindrical modulations have been tested, while the reflections occurring on the walls between two modulations are always diffuse.

For diffuse conditions on the cylinders, the TC of the $\mathrm{m}-\mathrm{NW}$ is systematically lower or equal to the membrane's one, but the two conductivities merge when the neck becomes smaller than $100 \mathrm{~nm}$. The same behavior is observed with specular reflections on the cylinders: $k_{m-N W} \leq k_{n p-m e m b}$ and the two curves converge when the neck is reduced. This means that the thermal transport is less hindered in NWs than in porous membranes with same characteristic dimensions surely because of the orientation of the scattering surfaces. Comparing figures 4 and 6 it is observed that both types of NW (with and without modulations) have lower TC than their equivalent membranes for neck sizes larger than $150 \mathrm{~nm}$.

In figure 7. results are plotted in terms of $S / \mathcal{V}$ ratio. For a same $S / \mathcal{V}$, the TC of the m-NW is more important than the membrane's one. This is coherent with the previous observation: in both systems, a part of the scattering surface is parallel to the measurement direction (fixed boundary conditions perpendicular to the $z$ axis), but in the $\mathrm{m}-\mathrm{NW}$ there is also the free surface between the cylinders perpendicular to the $y$ direction which is parallel to the $x$ axis. So a more important fraction of the scattering surface is parallel to the measurement direction in the m-NW. Therefore it is logic that the thermal transport is more reduced in the membrane for a same $S / \mathcal{V}$ ratio. When $S / \mathcal{V}$ increases $(i . e$ when the diameter increases), the difference in the TC between membranes and $\mathrm{m}-$ NW disappears, as the scattering surface perpendicular to the $y$ axis between the modulations is less and less influential.

The orientation of the scattering surfaces is an important parameter for the TC, this is also retrieved by Molecular Dynamics as it will be shown at the following. A m-NW with dimensions comparable to those of the systems studied in the section 4.1 has been modeled with MD. The maximum width of the m-NW 380 was set to $15 a_{0}$ and the diameter of the cylinders is $10 a_{0}$, so the neck is equal to $5 a_{0}$. Then this m-NW has to be compared with the membrane of the first line in the table $1\left(a=15 a_{0}, d=10 a_{0}\right.$ and $\left.n=a-d=5 a_{0}\right)$. With Molecular 
Dynamics, the TC of the m-NW is found to be $3.3 \pm 0.4 \mathrm{~W} / \mathrm{m} \mathrm{K}$ and its $S / \mathcal{V}$ ratio is equal to $0.77 \mathrm{~nm}^{-1}$. This is very close to the $\mathrm{TC}$ of the equivalent membrane $(3.4 \pm 0.3 \mathrm{~W} / \mathrm{m} \mathrm{K}$, see fig. 2). This definitely confirms that the difference between the TC of membranes and NWs is due to the orientation of the scattering surfaces compared to the direction of TC measurement. Moreover, the DOS of the nano-wires with and without modulations have been computed and the m-NW has exactly the same DOS as the equivalent membrane and the $5 a_{0}$ width NW. With these results, we can conclude that phononic effects do not dominate the TC of these systems at room temperature, and the reduction of the TC is due to the phonon scattering at the surfaces of the nano-structures.

\section{Conclusion}

This work provides insights about the thermal conductivity (TC) of phononiclike membranes and nano-wires with and without width modulations. Experimental results of Anufriev et al [57] have been retrieved and observations of Yu et al [1] are confirmed with two independent and complementary numerical techniques. The difference between the TC of periodic porous membranes and nano-wires' one is explained with geometric considerations by modeling an equivalent modulated nano-wire. In this system, a part of the scattering surface is perpendicular to the direction of measurement, as in the porous membrane. This leads to a reduced TC compared to a simple nano-wire, proving that the orientation of the scattering surface is important.

It is shown that the phononic effects in the periodic membrane do not exist at $300 \mathrm{~K}$. The surface's orientation is enough to explain the lower TC of the porous membrane compared to the nano-wire's one for most of the systems. Ultra-low TCs observed by Yu et al [2] concern nano-structured Silicon for temperature comprised between 100 and $300 \mathrm{~K}$. The current work achieved at room temperature does not show any coherent effect on the studied structures. Further investigation at lower temperatures is necessary to verify possible "phononic" behavior for the origin of the TC lowering. 
Finally, even if phononic effects are negligible, the $S / \mathcal{V}$ ratio is not sufficient to describe the thermal behavior of nano-structured materials because it does not take into account the scattering surface's geometry. A generalization of the TC appraisal in such nano-structures could possibly be achieved by introducing correction factors defining the orientation of the different scattering surfaces in the $S / \mathcal{V}$ parameter.

\section{Acknowledgements}

Authors would like to acknowledge to Mr. R. Jucquin (Lorraine University), Dr. A. France-Lanord (CEA Material Design), Dr. E. Lampin and Dr. J.F. Robillard (IEMN) for support and for many helpful discussions throughout this study. Calculations were partly performed on the large computer facilities GENCI-IDRIS (Grant 2014-x2014097186) and on the Ermione cluster (IJL-LEMTA).

\section{Supplementary materials}

In this section we present the TCs calculated with $\mathrm{MC}$ of nano-porous membranes of different thicknesses ( $80 \mathrm{~nm}$, fig. 8, and $145 \mathrm{~nm}$, fig. 9) and periodicities $(160 \mathrm{~nm}, 200 \mathrm{~nm}, 240 \mathrm{~nm}$ and $280 \mathrm{~nm})$ as a function of the neck. The TCs of equivalent nano-wires with height of $80 \mathrm{~nm}$ and $145 \mathrm{~nm}$ have been computed too for the sake of comparison. Then the critical neck size $n_{c}$, corresponding to the crossing of the curve of the membrane with the nano-wire's one, has been extracted for each periodicity and thickness. When the neck is smaller than $n_{c}$, the TC of the nano-wire is larger than the membrane's one. When the neck is smaller than $n_{c}$, the membrane has a larger TC than the nano-wire. The critical neck values $n_{c}$ for all configurations can be found in table 2

\section{References}

[1] Masahiro Nomura, Junki Nakagawa, Yuta Kage, Jeremie Maire, Dominik Moser, and Oliver Paul. Thermal phonon transport in silicon nanowires and 
two-dimensional phononic crystal nanostructures. Applied Physics Letters, 106(14):143102, April 2015.

[2] Jen-Kan Yu, Slobodan Mitrovic, Douglas Tham, Joseph Varghese, and James R. Heath. Reduction of thermal conductivity in phononic nanomesh structures. Nature Nanotechnology, 5(10):718-721, October 2010.

[3] A. G. Cullis, L. T. Canham, and P. D. J. Calcott. The structural and luminescence properties of porous silicon. Journal of Applied Physics, 82(3):909, 1997.

[4] Petra Granitzer and Klemens Rumpf. Porous SiliconA Versatile Host Material. Materials, 3(2):943-998, February 2010.

[5] Woochul Kim, Robert Wang, and Arun Majumdar. Nanostructuring expands thermal limits. Nano Today, 2(1):40 - 47, 2007.

[6] David G. Cahill, Wayne K. Ford, Kenneth E. Goodson, Gerald D. Mahan, Arun Majumdar, Humphrey J. Maris, Roberto Merlin, and Simon R. Phillpot. Nanoscale thermal transport. Journal of Applied Physics, 93(2):793, 2003.

[7] David G. Cahill, Paul V. Braun, Gang Chen, David R. Clarke, Shanhui Fan, Kenneth E. Goodson, Pawel Keblinski, William P. King, Gerald D. Mahan, Arun Majumdar, Humphrey J. Maris, Simon R. Phillpot, Eric Pop, and Li Shi. Nanoscale thermal transport. II. 20032012. Applied Physics Reviews, 1(1):-, 2014.

460

[8] L. D. Hicks, T. C. Harman, and M. S. Dresselhaus. Use of quantumwell superlattices to obtain a high figure of merit from nonconventional thermoelectric materials. Applied Physics Letters, 63(23):3230-3232, 1993.

[9] Arun Majumdar. Thermoelectricity in Semiconductor Nanostructures. Science, 303(5659):777-778, 2004. 
[10] David L. Andrews and Gregory D. Scholes, editors. Comprehensive nanoscience and technology. Elsevier, Acad. Press, Amsterdam, 2011.

[11] John M. Ziman. Electrons and phonons: the theory of transport phenomena in solids. Oxford classic texts in the physical sciences. Clarendon Press, Oxford, 2007. OCLC: 838077875.

[12] James Clerk Maxwell. A Treatise on Electricity and Magnetism. Cambridge University Press, Cambridge, 1873.

[13] Lord Rayleigh. LVI. On the influence of obstacles arranged in rectangular order upon the properties of a medium. Philosophical Magazine Series 5, 34(211):481-502, December 1892.

[14] D. P. H. Hasselman and Lloyd F. Johnson. Effective thermal conductivity of composites with interfacial thermal barrier resistance. Journal of Composite Materials, 21(6):508-515, 1987.

[15] Ce-Wen Nan, R. Birringer, David R. Clarke, and H. Gleiter. Effective thermal conductivity of particulate composites with interfacial thermal resistance. Journal of Applied Physics, 81(10):6692, 1997.

[16] R.H. Tarkhanyan and D.G. Niarchos. Reduction in lattice thermal conductivity of porous materials due to inhomogeneous porosity. International Journal of Thermal Sciences, 67:107-112, May 2013.

[17] G. Chen. Thermal conductivity and ballistic-phonon transport in the crossplane direction of superlattices. Phys. Rev. B, 57(23):14958-14973, June 1998.

[18] B. C. Daly, Maris, Y. H. J. Tanaka, and S. Tamura. Molecular dynamics calculation of the In-plane thermal conductivity of GaAs/AlAs superlattices. Phys. Rev. B, 67(3):033308, January 2003.

490

[19] S. M. Lee, D. G. Cahill, and R. Venkatasubramanian. Thermal conductivity of SiGe superlattices. Applied Physics Letters, 70(22):2957-2959, 1997. 
[20] R. Venkatasubramanian. Thin-film thermoelectric devices with high roomtemperature figures of merit. Nature, 413:597-602, October 2001.

[21] Sanghamitra Neogi, J. Sebastian Reparaz, Luiz Felipe C. Pereira, Bartlomiej Graczykowski, Markus R. Wagner, Marianna Sledzinska, Andrey Shchepetov, Mika Prunnila, Jouni Ahopelto, Clivia M. Sotomayor-Torres, and Davide Donadio. Tuning Thermal Transport in Ultrathin Silicon Membranes by Surface Nanoscale Engineering. ACS Nano, 9(4):3820-3828, April 2015.

[22] Basil T. Wong, Mathieu Francoeur, and M. Pinar Meng. A Monte Carlo simulation for phonon transport within silicon structures at nanoscales with heat generation. International Journal of Heat and Mass Transfer, 54(910):1825-1838, April 2011.

[23] Ruikang $\mathrm{Wu}$, Run $\mathrm{Hu}$, and Xiaobing Luo. First-principle-based fulldispersion Monte Carlo simulation of the anisotropic phonon transport in the wurtzite GaN thin film. Journal of Applied Physics, 119(14):145706, April 2016.

[24] V. Jean, S. Fumeron, K. Termentzidis, X. Zianni, and D. Lacroix. Monte Carlo simulations of phonon transport in Si nanowires with constrictions. International Journal of Heat and Mass Transfer, 86:648 - 655, 2015.

[25] Davide Donadio and Giulia Galli. Temperature Dependence of the Thermal Conductivity of Thin Silicon Nanowires. Nano Letters, 10(3):847-851, March 2010.

[26] Yuping He and Giulia Galli. Microscopic Origin of the Reduced Thermal 515 Conductivity of Silicon Nanowires. Phys. Rev. Lett., 108(21):215901, May 2012.

[27] Deyu Li, Yiying Wu, Philip Kim, Li Shi, Peidong Yang, and Arun Majumdar. Thermal conductivity of individual silicon nanowires. Applied Physics Letters, 83(14):2934-2936, 2003. 
[28] Arden L. Moore, Sanjoy K. Saha, Ravi S. Prasher, and Li Shi. nanowires. Applied Physics Letters, 93(8):083112, 2008.

[29] X. Cartoixà, R. Dettori, C. Melis, L. Colombo, R. Rurali, Thermal transport in porous Si nanowires from approach-to-equilibrium molecular dynamics calculations Applied Physics Letters 109 (1) 013107, 2016.

[30] Riccardo Dettori, Claudio Melis, Xavier Cartoix, Riccardo Rurali, and Luciano Colombo. Model for thermal conductivity in nanoporous silicon from atomistic simulations. Phys. Rev. B, 91(5):054305, February 2015.

[31] Jin Fang and Laurent Pilon. Scaling laws for thermal conductivity of crystalline nanoporous silicon based on molecular dynamics simulations. Journal of Applied Physics, 110(6):064305, 2011.

[32] Yuping He, Davide Donadio, and Giulia Galli. Morphology and Temperature Dependence of the Thermal Conductivity of Nanoporous SiGe. Nano Letters, 11(9):3608-3611, September 2011.

${ }_{535}$ [33] V. Jean, S. Fumeron, K. Termentzidis, S. Tutashkonko, and D. Lacroix. Monte Carlo simulations of phonon transport in nanoporous silicon and germanium. Journal of Applied Physics, 115(2):024304, January 2014.

[34] V. Lacatena, M. Haras, J.-F. Robillard, S. Monfray, T. Skotnicki, and E. Dubois. Phononic engineering of silicon using dots on the fly e-beam lithography and plasma etching. Microelectronic Engineering, 121:131-134, June 2014.

[35] V. Lacatena, M. Haras, J.-F. Robillard, S. Monfray, T. Skotnicki, and E. Dubois. Toward quantitative modeling of silicon phononic thermocrystals. Applied Physics Letters, 106(11):114104, March 2015.

${ }_{545}$ [36] Patrick E. Hopkins, Charles M. Reinke, Mehmet F. Su, Roy H. Olsson, Eric A. Shaner, Zayd C. Leseman, Justin R. Serrano, Leslie M. Phinney, 
and Ihab El-Kady. Reduction in the Thermal Conductivity of Single Crystalline Silicon by Phononic Crystal Patterning. Nano Letters, 11(1):107112, January 2011.

[37] Jaeho Lee, Jongwoo Lim, and Peidong Yang. Ballistic Phonon Transport in Holey Silicon. Nano Letters, 15(5):3273-3279, 2015.

[38] David Song and Gang Chen. Thermal conductivity of periodic microporous silicon films. Applied Physics Letters, 84(5):687, 2004.

[39] Eli Yablonovitch. Photonic band-gap structures. JOSA B, 10(2):283-295, 1993.

[40] Alvaro Blanco, Emmanuel Chomski, Serguei Grabtchak, Marta Ibisate, Sajeev John, Stephen Leonard, Cefe Lopez, Francisco Meseguer, Hernan Miguez, Jessica Mondia, Geoffrey Ozin, Ovidiu Toader, and Henry Van Driel. Large-scale synthesis of a silicon photonic crystal with a complete three-dimensional bandgap near 1.5 micrometres. Nature, 405:437440, 2000.

[41] Nobuyuki Zen, Tuomas A. Puurtinen, Tero J. Isotalo, Saumyadip Chaudhuri, and Ilari J. Maasilta. Engineering thermal conductance using a twodimensional phononic crystal. Nature Communications, 5, March 2014.

[42] Junki Nakagawa, Yuta Kage, Takuma Hori, Junichiro Shiomi, and Masahiro Nomura. Crystal structure dependent thermal conductivity in two-dimensional phononic crystal nanostructures. Applied Physics Letters, 107(2):023104, July 2015.

[43] Lina Yang, Nuo Yang, and Baowen Li. Extreme Low Thermal Conductivity in Nanoscale 3d Si Phononic Crystal with Spherical Pores. Nano Letters, 14(4):1734-1738, April 2014.

[44] Lina Yang, Nuo Yang, and Baowen Li. Reduction of Thermal Conductivity by Nanoscale 3d Phononic Crystal. Scientific Reports, 3, January 2013. 
[45] P. E. Hopkins, L. M. Phinney, P. T. Rakich, R. H. Olsson, and I. El-Kady. Phonon considerations in the reduction of thermal conductivity in phononic crystals. Applied Physics A, 103(3):575-579, June 2011.

[46] LAMMPS Molecular Dynamics Simulator.

[47] Denis J. Evans and Gary P. Morriss. Statistical mechanics of nonequilibrium liquids. ANU E Press, Canberra, [2nd] ed edition, 2007.

[48] Frank H. Stillinger and Thomas A. Weber. Computer simulation of local order in condensed phases of silicon. Physical Review B, 31(8):5262-5271, April 1985.

[49] R.L.C. Vink, G.T. Barkema, W.F. van der Weg, and Normand Mousseau. Fitting the StillingerWeber potential to amorphous silicon. Journal of NonCrystalline Solids, 282(2-3):248-255, April 2001.

[50] M. Verdier, K. Termentzidis, and D. Lacroix. Crystalline-amorphous silicon nano-composites: Nano-pores and nano-inclusions impact on the thermal conductivity. Journal of Applied Physics, 119(17):175104, May 2016.

[51] C. J. Glassbrenner and Glen A. Slack. Thermal conductivity of silicon and germanium from $3 \mathrm{~K}$ to the melting point. Physical Review, 134(4A):A1058, 1964.

[52] P.D. Maycock. Thermal conductivity of Silicon, Germanium, III-V compounds and III-V alloys. Solid-State Electronics, 10:161-168, 1967.

[53] William Humphrey, Andrew Dalke, and Klaus Schulten. VMD - Visual Molecular Dynamics. Journal of Molecular Graphics, 14:33-38, 1996.

[54] M. G. Holland. Phonon Scattering in Semiconductors From Thermal Conductivity Studies. Phys. Rev., 134(2A):A471-A480, April 1964.

[55] JEAN Valentin. Modlisation du transport de phonons dans les semiconducteurs nanostructurs. 
[56] B. Fu, G. H. Tang, and C. Bi. Thermal conductivity in nanostructured materials and analysis of local angle between heat fluxes. Journal of Applied Physics, 116(12):124310, September 2014.

[57] Roman Anufriev, Jeremie Maire, and Masahiro Nomura. Reduction of thermal conductivity by surface scattering of phonons in periodic silicon nanostructures. Physical Review B, 93(4):045411, January 2016.

[58] Yong Fu Zhu, Jian She Lian, and Qing Jiang. Re-examination of Casimir limit for phonon traveling in semiconductor nanostructures. Applied Physics Letters, 92:113101, 2008.

610

[59] R. Prasher. Predicting the Thermal Resistance of Nanosized Constrictions. Nano Letters, 5(11):2155-2159, 2005.

[60] Sebastian Volz, and Pierre-Olivier Chapuis. Increase of thermal resistance 615 between a nanostructure and a surface due to phonon multireflections. Journal of Applied Physics, 103(3):034306, 2008.

[61] Zhiting Tian, Keivan Esfarjani, Junichiro Shiomi, Asegun S. Henry, and Gang Chen. On the importance of optical phonons to thermal conductivity in nanostructures. Applied Physics Letters, 99(5):053122, 2011. 

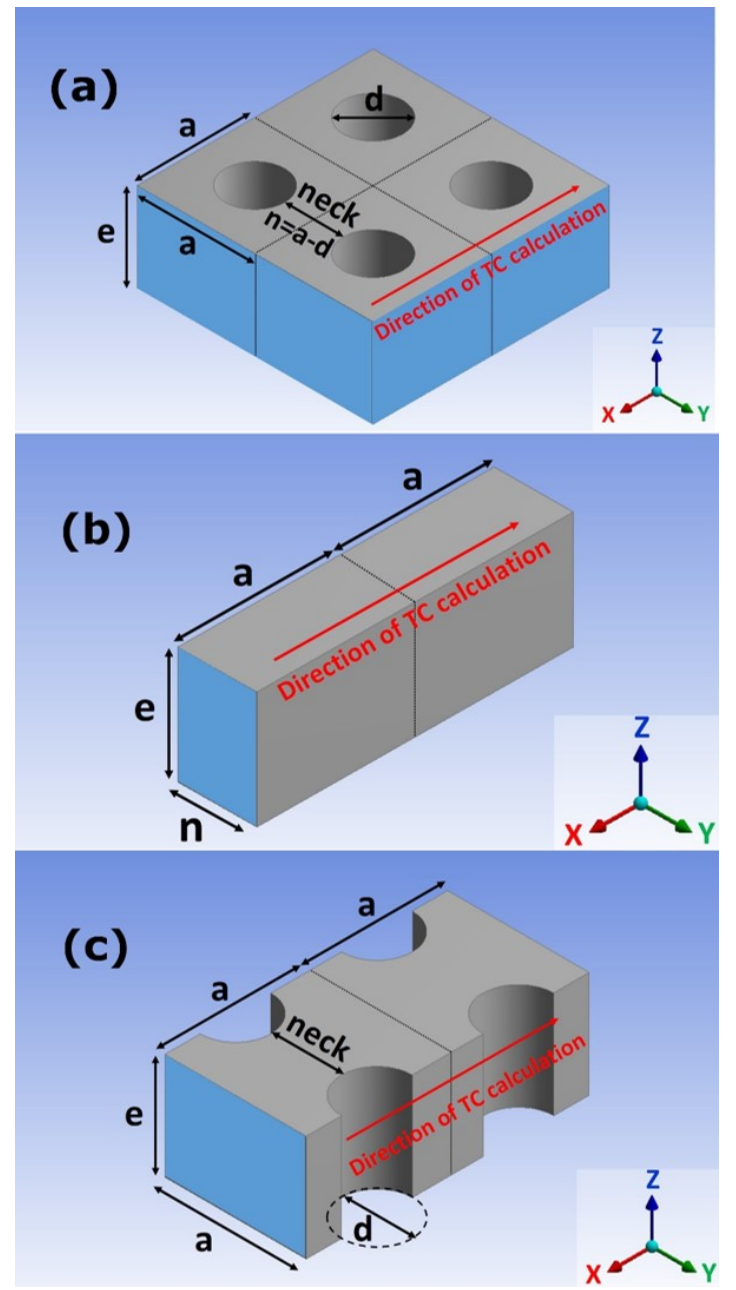

Figure 1: Schematic representation of the modeled systems. (a) phononic-like nano-porous membranes (np-memb). Blue faces indicate periodic boundary conditions in these directions, the other direction is not periodic as it is the direction of the thickness of the membrane. (b) equivalent nano-wire (NW) whose width is equal to the neck between two adjacent pores in the porous membrane. The height is equal to the thickness of the membrane. The blue face indicates a periodic boundary condition in this direction (length of the nano-wire), the other directions are not periodic (walls of the nano-wire). (c) equivalent modulated nano-wire $(\mathrm{m}-\mathrm{NW})$. Its width is equal to the periodicity $a$ of the porous membrane and is modulated by periodic and cylindrical constrictions with the same diameter $d$ as the pores. Thus, the minimum width is equal to the neck between two neighborous pores in the membrane. The blue face indicates a periodic boundary condition in this direction (length of the m-NW), the other directions are not periodic (walls of the m-NW). 


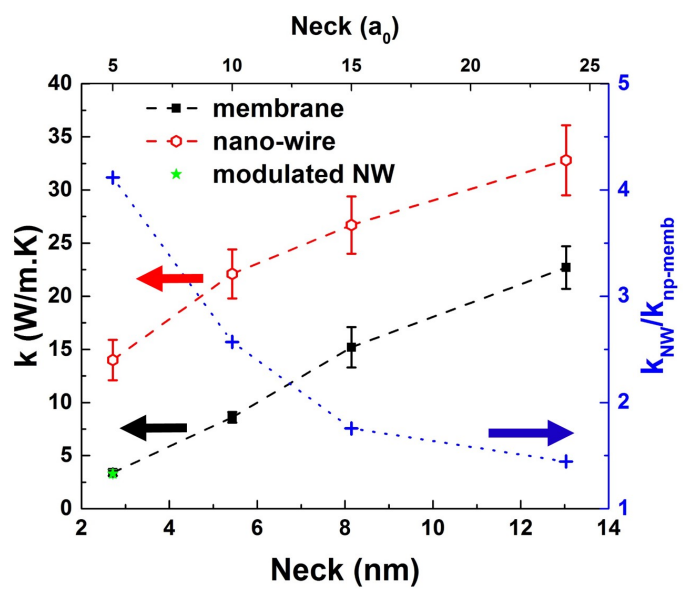

Figure 2: TC (computed by MD) of porous membranes and equivalent NWs versus neck size, calculated with EMD (left axis). The ratio of the TC of the NW to membrane's one is given for each neck size (right axis).

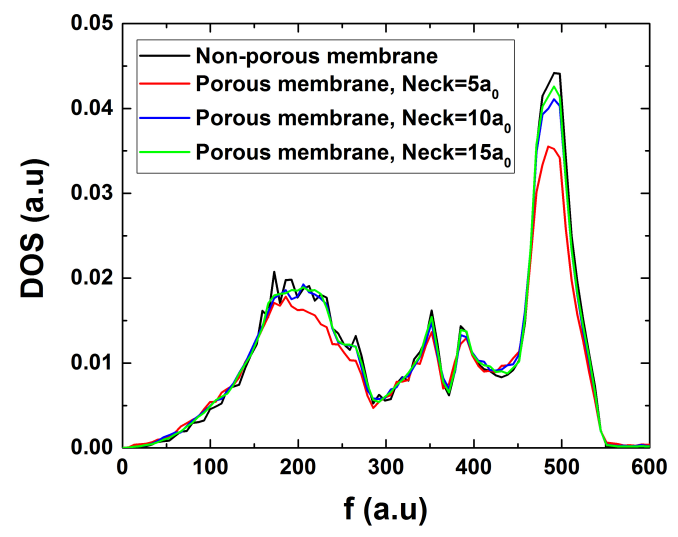

Figure 3: Phonon Density of State (DOS) of the nano-porous membranes compared to the DOS of a membrane of same thickness without pore. Modifications in phonons populations due to the porosity do not seem to be important for thermal transport. 


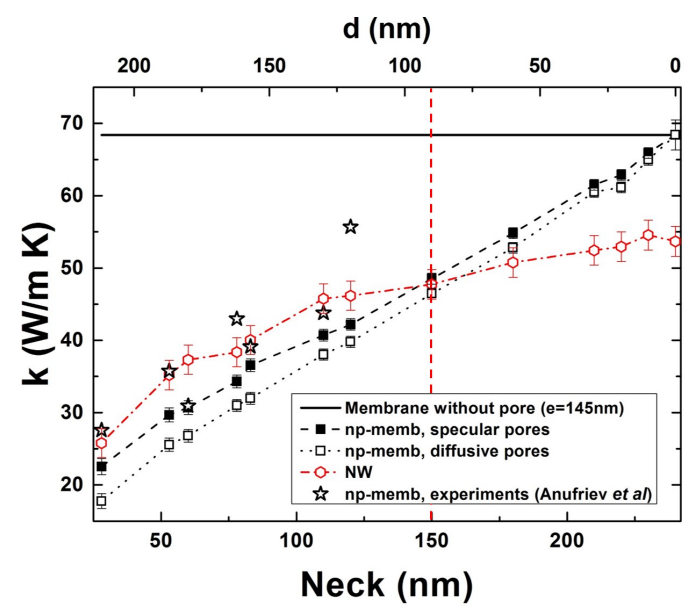

Figure 4: TCs (calculated by MC) of nano-porous membranes and equivalent NWs in terms of the neck size. When the latter is small, the TC of the NW is once more greater than the membrane's TC. Experimental results for membranes with the same geometric configuration can be found in ref [57.

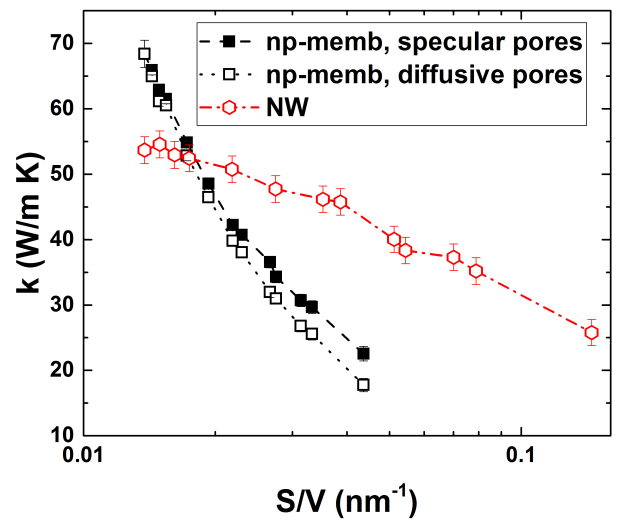

Figure 5: TCs (calculated by MC) of nano-porous membranes and equivalent NWs in terms of the $S / \mathcal{V}$ ratio. 


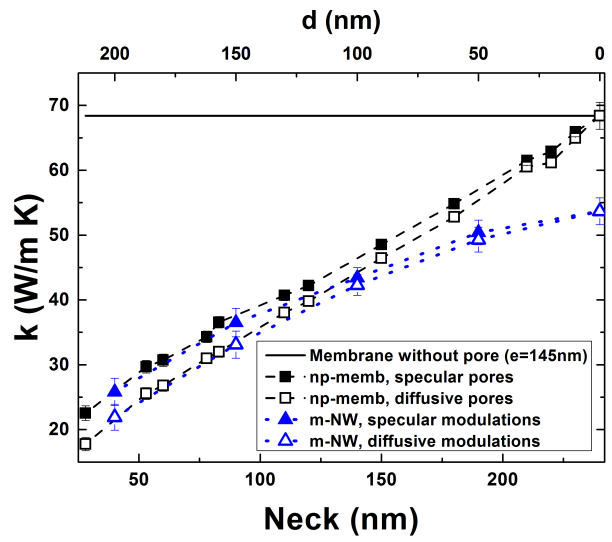

Figure 6: TCs computed with Monte Carlo of nanoporous membranes (np-memb) and equivalent modulated nano-wires (m-NW) in terms of the neck size.

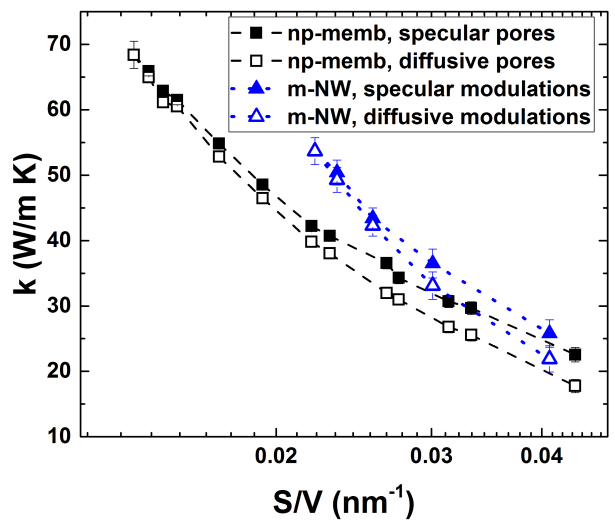

Figure 7: TCs computed with Monte Carlo of nanoporous membranes and equivalent m-NWs in terms of the $S / \mathcal{V}$ ratio. 


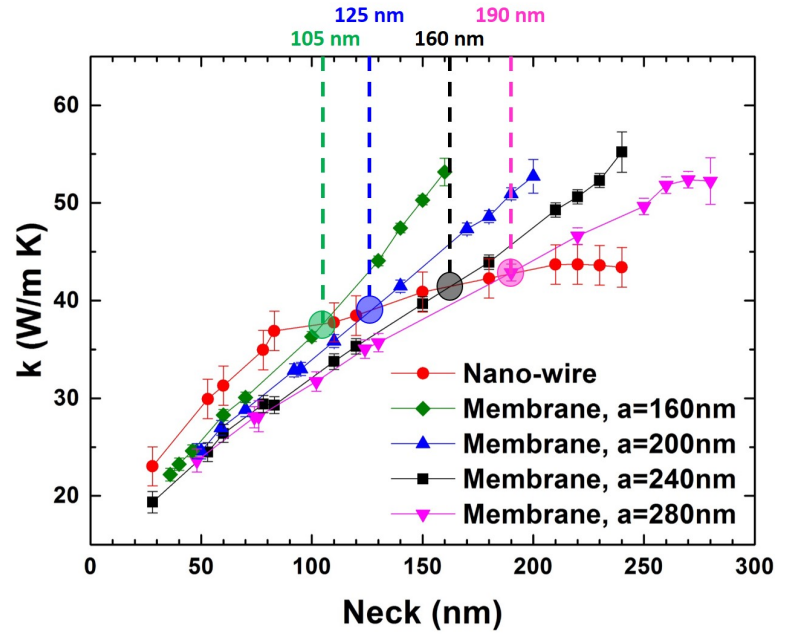

Figure 8: TCs (computed by MC) of membranes with thickness of $80 \mathrm{~nm}$ for various periodicities compared to the TC of the nano-wire with height of $80 \mathrm{~nm}$. The values of the critical neck size $n_{c}$ for which membrane's TC is equal to NW's one can be found in table 2

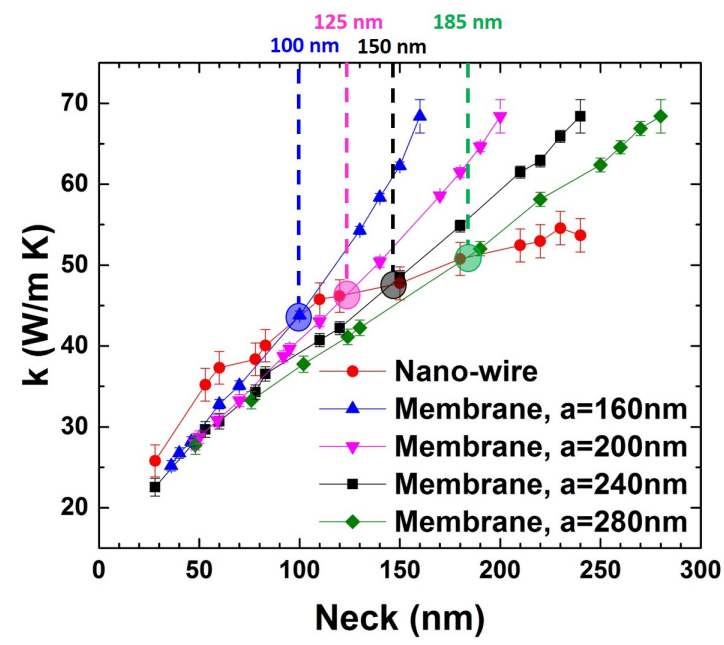

Figure 9: TCs (computed by MC) of membranes with thickness of $145 \mathrm{~nm}$ for various periodicities compared to the TC of a nano-wire with height of $145 \mathrm{~nm}$. The values of the critical neck size $n_{c}$ for which membrane's TC is equal to NW's one can be found in table 2 